\title{
Neurology and psychiatry: waking up to opportunities of sleep. State of the art and clinical/research priorities for the next decade
}

\author{
C. L. Bassetti ${ }^{1}$, L. Ferini-Strambi ${ }^{2}$, S. Brown ${ }^{3}$, A. Adamantidis ${ }^{1}$, F. Benedetti $^{4}$, O. Bruni ${ }^{5}$, C. Cajochen ${ }^{6}$, \\ L. Dolenc-Groseli ${ }^{7}$, R. Ferri ${ }^{8}$, S. Gais ${ }^{9}$, R. Huber ${ }^{10}$, R. Khatami ${ }^{11}$, G. J. Lammers ${ }^{12,13}$, P. H. Luppi ${ }^{14}$ \\ M. Manconi ${ }^{15}$, C. Nissen ${ }^{16}$, L. Nobili ${ }^{17}$, P. Peigneux ${ }^{18}$, T. Pollmächer $^{19}$, W. Randerath ${ }^{20}$, D. Riemann ${ }^{16}$ \\ J. Santamaria ${ }^{21}$, K. Schindler ${ }^{1}$, M. Tafti ${ }^{22,23}$, E. Van Someren ${ }^{24,25}$ and T. C. Wetter ${ }^{26}$
}

\begin{abstract}
${ }^{1}$ Department of Neurology, Inselspital, Bern University Hospital, University of Bern, Bern, Switzerland; ${ }^{2}$ Division of Neuroscience, Sleep Disorders Centre, Università Vita-Salute San Raffaele, Milan, Italy, ${ }^{3}$ Institute of Pharmacology and Toxicology, University of Zürich, Zürich, Switzerland; ${ }^{4}$ Department of Clinical Neurosciences, Scientific Institute and University Vita-Salute San Raffaele, Milan; ${ }^{5}$ Department of Developmental and Social Psychology, Sapienza University, Rome, Italy; ${ }^{6}$ Psychiatric University Clinic, Basel, Switzerland; ${ }^{7}$ Division of Neurology, Institute of Clinical Neurophysiology, University Medical Centre Ljubljana, Ljubljana, Slovenia; ${ }^{8}$ Department of Neurology, Oasi Institute for Research on Mental Retardation and Brain Aging (IRCCS), Troina, Italy; ${ }^{9}$ Medical Psychology and Behavioural Neurobiology, Eberhard Karls Universität Tübingen, Tübingen, Germany; ${ }^{10}$ Department of Paediatrics, Children's University Hospital, Zurich; ${ }^{11}$ Sleep

Centre, Klinik Barmelweid AG, Barmelweid, Switzerland; ${ }^{12}$ Department of Neurology and Clinical Neurophysiology, Leiden University Medical Centre, Leiden; ${ }^{13}$ Sleep Wake Centre SEIN, Stichting Epilepsie Instellingen Nederland, Heemstede, The Netherlands; ${ }^{14}$ UMR 5292

CNRS/U1028 INSERM, Centre de Recherche en Neurosciences de Lyon (CRNL), Team "Physiopathologie des réseaux neuronaux responsables du cycle veille-sommeil”, Université Claude Bernard Lyon I, Lyon, France; ${ }^{15}$ Sleep and Epilepsy Centre, Neurocentre of Southern Switzerland, Civic Hospital (EOC) of Lugano, Lugano, Switzerland; ${ }^{16}$ Department of Clinical Psychology and Psychophysiology/Sleep Medicine, Centre for Mental Disorders, Freiburg University Medical Centre, Freiburg, Germany; ${ }^{17}$ Centre of Epilepsy Surgery 'C. Munari', Niguarda Hospital, Milan, Italy; ${ }^{18}$ UR2NF - Neuropsychology and Functional Neuroimaging Research Unit, CRCN - Centre de Recherches Cognition et Neurosciences and UNI - ULB Neurosciences Institute, Université Libre de Bruxelles (ULB), Brussels, Belgium; ${ }^{19}$ Center of Mental Health, Klinikum Ingolstadt, Ingolstadt; ${ }^{20}$ Institut für Pneumologie, Krankenhaus Bethanien gGmbH, Universität Witten/Herdecke, Solingen, Germany; ${ }^{21}$ Neurology Service, Hospital Clínic of Barcelona, Institut d'Investigació Biomèdiques August Pi i Sunyer (IDIBAPS),

Centro de Investigación Biomédica en Red sobre Enfermedades Neurodegenerativas (CIBERNED), Barcelona, Spain; ${ }^{22}$ Centre for

Integrative Genomics, University of Lausanne, , ${ }^{23}$ Centre for Investigation and Research in Sleep, Vaud University Hospital, Lausanne, Switzerland; ${ }^{24}$ Department of Sleep and Cognition, Netherlands Institute for Neuroscience, ; ${ }^{25}$ Departments of Integrative Neurophysiology and Medical Psychology, Center for Neurogenomics and Cognitive Research (CNCR), VU University and Medical Center, Amsterdam, The Netherlands; and ${ }^{26}$ Department of Psychiatry and Psychotherapy, University of Regensburg, Regensburg, Germany
\end{abstract}

\section{Keywords: \\ neurological disorders, neurology, psychiatric disorders, psychiatry, sleep research, sleep, sleep-wake disorders}

Received 1 June 2015 Accepted 5 June 2015

European Journal of Neurology 2015, 22: 13371354

doi:10.1111/ene.12781
In recent years, evidence has emerged for a bidirectional relationship between sleep and neurological and psychiatric disorders. First, sleep-wake disorders (SWDs) are very common and may be the first/main manifestation of underlying neurological and psychiatric disorders. Secondly, SWDs may represent an independent risk factor for neuropsychiatric morbidities. Thirdly, sleep-wake function (SWF) may influence the course and outcome of neurological and psychiatric disorders. This review summarizes the most important research and clinical findings in the fields of neuropsychiatric sleep and circadian research and medicine, and discusses the promise they bear for the next decade. The findings herein summarize discussions conducted in a workshop with 26 European experts in these fields, and formulate specific future priorities for clinical practice and translational research. More generally, the conclusion emerging from this workshop is the recognition of a tremendous opportunity offered by our knowledge of SWF and SWDs that has unfortunately not yet entered as an important key factor in clinical practice, particularly in Europe. Strengthening pre-graduate and postgraduate teaching, creating academic multidisciplinary sleep-wake centres and simplifying diagnostic approaches of SWDs coupled with targeted treatment strategies yield enormous clinical benefits for these diseases.

Correspondence: C. L. Bassetti, Department of Neurology, Inselspital, Bern University Hospital, University of Bern, Bern, Switzerland (tel.: +41 (0) 316323066; fax: + 41 (0) 316329679; e-mail: claudio.bassetti@insel.ch). L. Ferini-Strambi, Sleep Disorders Centre, Division of Neuroscience, Università Vita-Salute San Raffaele, Milan, Italy (tel.: + 39 (02) 2643 3363; fax: + 39 (02) 2643 3394; e-mail: ferinistrambi.luigi@ hsr.it). Organized by the scientific committee of the Alpine Sleep Summer School (ASSS); Endorsed by the European Sleep Research Society (ESRS); European Academy of Neurology (EAN); European Psychiatric Association (EPA); European Society of Biological Psychiatry (ESBP) 


\section{Introduction}

The last decade has brought exciting research findings and medical insights that demonstrate the key involvement of sleep in several neurological and psychiatric disorders.

These main achievements can be summarized in the following five domains.

1 Epidemiology: Sleep-wake disturbances (SWDs) are extremely prevalent in patients with neurological and psychiatric (Table 1) disorders [1]. This very frequent comorbidity is linked to the neurobiology of sleep, psychiatric and neurological functions. Mechanistically, this comorbidity is probably based on overlapping neuronal networks and common neurotransmitter systems (Fig. 1a-c).

2 Diagnostic value: SWDs represent an independent risk factor for neurological and psychiatric disorders. Sleep-disordered breathing (SDB), for example, was shown to be an independent predictor for stroke [2]. Detrimental effects of SDB, and possibly also of insomnia and restless legs syndrome (RLS), upon cardiovascular functions have been documented and recently linked with data on increased long-term cardiovascular morbidity and mortality [3-5] (Fig. 2). Furthermore, SWDs may be the first or main manifestation of neurological and psychiatric disorders. For example, rapid eye movement (REM) sleep behaviour disorder (RBD) is most commonly symptomatic of an underlying neurodegenerative disorder [6]. Insomnia can be the first manifestation of depression; and chronic insomnia of unknown origin is significantly associated with an increased risk for depression (and eventually suicidality) [7]. Hypersomnia/excessive daytime sleepiness (EDS) can be amongst the first manifestations of parkinsonism [8]. Subtle disturbances of circadian rhythms are present in early stages of Alzheimer's disease [9] whilst clinically evident disturbances of circadian rhythms and/or modulation of neuropsychiatric functions are a common feature of seasonal affective disorder, late stage Alzheimer's disease, Parkinson's disease and psychiatric disorders such as major depressive disorder, bipolar disorder and schizophrenia [10].

3 Treatment and prognosis: SWDs may have a detrimental effect on the course of different neurological and psychiatric disorders. SDB, for example,

Table 1 Frequency of sleep-wake disturbances (SWDs) in patients with neurological and psychiatric disorders

\begin{tabular}{|c|c|c|c|c|c|c|c|}
\hline & Insomnia & EDS-H-F & SDB & RLS & Parasomnia & $\begin{array}{l}\text { Circadian } \\
\text { disease }\end{array}$ & Special features \\
\hline \multicolumn{8}{|l|}{ Neurology } \\
\hline Stroke & + & + & +++ & ++ & + & + & $\begin{array}{l}\text { Severe hypersomnia in } \\
\text { bithalamic lesion } \\
\text { Central sleep apnoea in } \\
\text { brainstem lesion }\end{array}$ \\
\hline Parkinsonism & $+/++^{\mathrm{a}}$ & $+/+++^{\mathrm{a}}$ & ++ & ++ & $+++^{\mathrm{b}}$ & ++ & $\begin{array}{l}\text { Stridor in multiple system atrophy } \\
\text { (Shy-Drager variant) }\end{array}$ \\
\hline Dementia & ++ & ++ & + & + & ++ & +++ & Sundowning \\
\hline Epilepsy & + & ++ & + & + & ++ & + & Nocturnal frontal lobe epilepsy \\
\hline Headache & ++ & + & $++^{c}$ & ++ & + & + & $\begin{array}{l}\text { Hypnic headache } \\
\text { Exploding head syndrome }\end{array}$ \\
\hline Multiple sclerosis & $++^{\mathrm{d}}$ & ++ & + & ++ & + & + & Daytime F \\
\hline Neuromuscular dis & + & ++ & +++ & + & + & + & $\mathrm{H}$ in myotonia Steinert \\
\hline Head trauma & ++ & +++ & + & + & + & + & \\
\hline \multicolumn{8}{|l|}{ Psychiatry } \\
\hline Anxiety & $+++^{\mathrm{e}}$ & $+/++^{\mathrm{f}}$ & + & + & + & + & Nocturnal panic attacks \\
\hline Depression & $+++^{\mathrm{g}}$ & $+/++^{\mathrm{h}}$ & ++ & $++^{\mathrm{h}}$ & + & $++^{\mathrm{i}}$ & \\
\hline Bipolar disorder & $+++^{j}$ & + & + & + & + & + & \\
\hline Schizophrenia & ++ & ++ & + & + & + & $+++^{\mathrm{k}, 1}$ & \\
\hline Drug/substance abuse & $+++^{\mathrm{m}}$ & $++/+++^{\mathrm{n}}$ & $++^{\circ}$ & + & $++^{\mathrm{p}}$ & + & Withdrawal symptoms \\
\hline
\end{tabular}

Frequency of occurrence: + low (similar to general population or slightly more frequent); ++ moderate (higher than in general population); +++ high (at least $50 \%$ of patients). EDS, excessive daytime sleepiness; H, hypersomnia; F, fatigue; RLS, restless legs syndrome; SDB, sleep-disordered breathing. ${ }^{a}$ In advanced stages/late course of the disease; ${ }^{b}$ rapid eye movement behaviour disorder; ${ }^{c}$ morning headache as a symptom of SDB, SDB triggering migraine/cluster headache; ${ }^{d}$ acute recurrent insomnia due to interferon and steroid treatments; ${ }^{e}$ especially difficulties in initiating sleep; ${ }^{\mathrm{f}}$ secondary to anxiolytic drugs; ${ }^{\mathrm{g}}$ especially maintenance insomnia with early morning awakenings; ${ }^{\mathrm{h}}$ secondary to antidepressant drugs; ${ }^{i}$ tendency to the advanced phase shift pattern; ${ }^{j}$ insomnia with restriction of time in bed preceding or during the maniac phase; ${ }^{\mathrm{k}}$ many variants of circadian rhythm disturbances due to reduced social interaction, isolation or medication; ${ }^{1}$ circadian rhythm disruption often precedes schizophrenia symptoms; ${ }^{\mathrm{m}}$ secondary to excitant substances/drugs; ${ }^{\mathrm{n}}$ secondary to sedative drugs; ${ }^{\mathrm{o}}$ central apnoeas secondary to opioids or exacerbations of obstructive sleep apnoeas secondary to sedative/benzodiazepine drugs; ${ }^{\mathrm{p}}$ confusional arousals due to drug medication. 
(a)

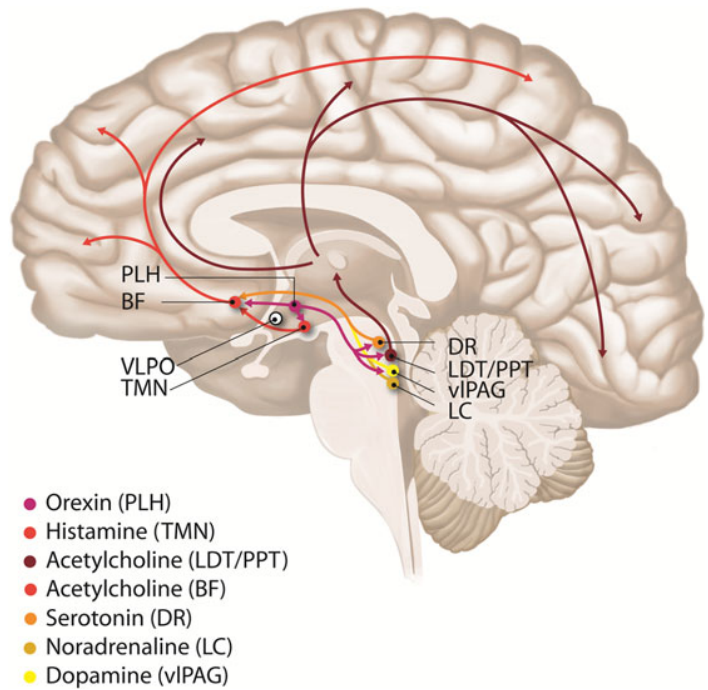

(b)

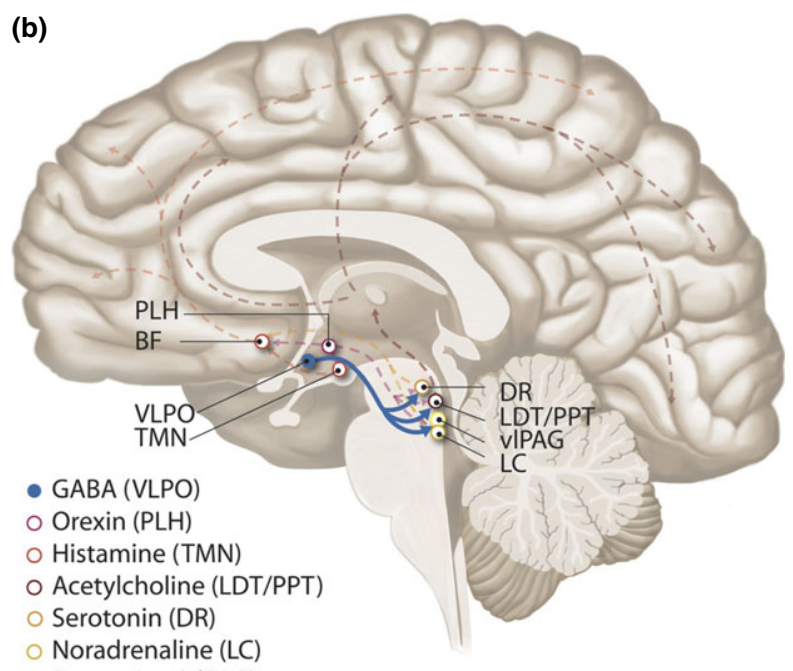

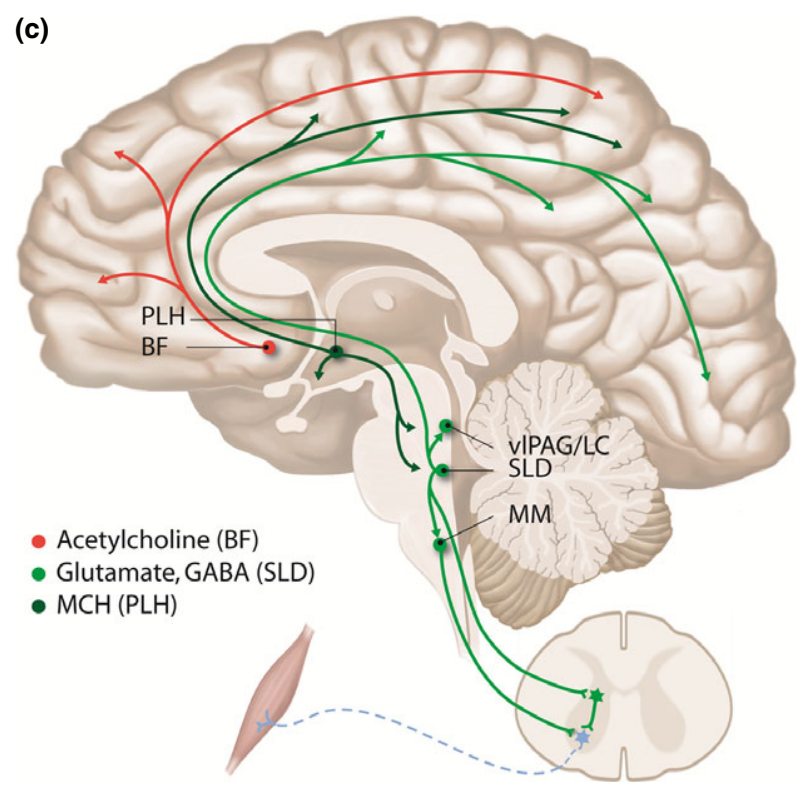

Figure 1 (a) Neuroanatomy and neurochemistry of sleep-wake functions (SWFs)/circuits (wakefulness). For details on specific functions/interactions of these neuronal networks see elsewhere [1,95]. Illustration by Anja Giger, Neurocenter, University Hospital, Inselspital, Bern, Switzerland. (b) Neuroanatomy and neurochemistry of SWFs/circuits (sleep onset/non-rapid-eye-movement sleep). For details on specific functions/interactions of these neuronal networks see elsewhere [1,95]. Illustration by Anja Giger, Neurocenter, University Hospital, Inselspital, Bern, Switzerland. (c) Neuroanatomy and neurochemistry of SWFs/circuits (REM sleep). For details on specific functions/interactions of these neuronal networks see elsewhere [1,95]. Illustration by Anja Giger, Neurocenter, University Hospital, Inselspital, Bern, Switzerland. PLH, posterior lateral hypothalamus; TMN, tubero-mamillary nucleus; LDT/PPT, laterodorsal tegmental nucleus/pedunculopontine tegmental nucleus; DR, dorsal raphe nucleus; LC, locus coeruleus; vlPAG, ventrolateral periaqueductal gray; VLPO, ventrolateral pre-optic nucleus; BF, basal forebrain; SLD, sublaterodorsal nucleus (just ventral to LC).

was shown to worsen the outcome of stroke and epilepsy and to affect cognition [11-13]. On the other hand, specific treatment of SWDs in neurological and psychiatric patients can have positive consequences on neuropsychiatric disease manifestations and progression, and eventually improve quality of life and survival. For example, treatment with continuous positive air pressure (CPAP) can improve seizure control in patients with epilepsy, whereas light and melatonin can ameliorate cognitive functions in dementia patients $[14,15]$. Finally, drugs used in neurological and psychiatric patients may cause or worsen SWDs, e.g. sleep attacks/ EDS or insomnia with dopaminergic drugs in patients with parkinsonism; RBD and RLS caused by antidepressants $[16,17]$. 

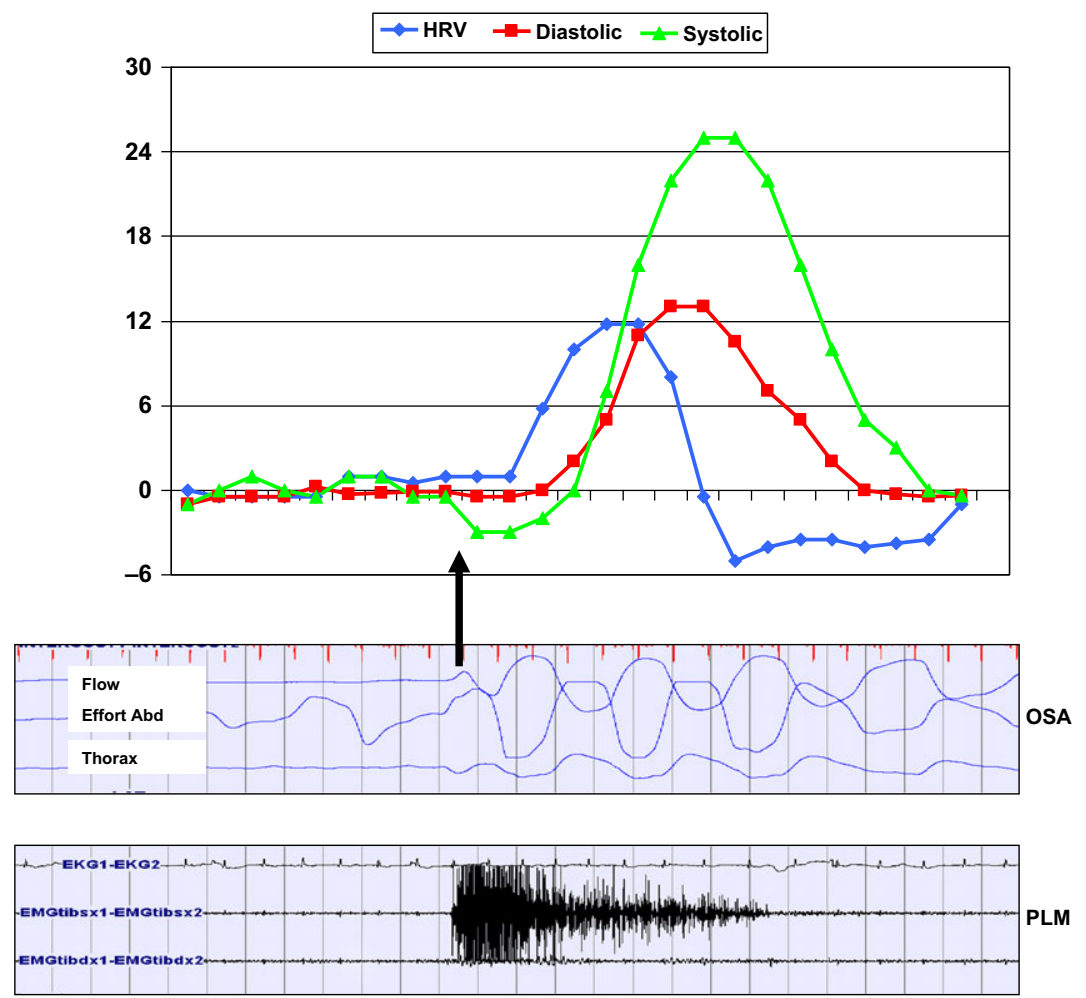

Figure 2 Periodic limb movements (PLMs) in sleep and obstructive sleep apnoea (OSA) events lead to similar arousal and cardiovascular responses [increase in heart rate variability (HRV), systolic and diastolic blood pressure], which in the long term increase cardiovascular and cerebrovascular morbidity (e.g. stroke) and mortality.
4 Neurobiological insights gained from the study of SWDs: recent clinical and translational sleep/circadian studies - including the development of a few animal models [18] - provided interesting new insights for the entire field of neurobiology. The discovery of an animal model of narcolepsy has enhanced our understanding of hypothalamic mechanisms of sleep-wake regulation, but also more generally of motor, autonomic and emotional functions in the healthy brain [1]. The observation of a disturbance of circadian functions in patients with seasonal affective disorder, Alzheimer's disease and bipolar disorders suggests a crucial involvement of chronobiological rhythms in the pathophysiology of these conditions [10]. Our understanding of RBD as an early manifestation of neurodegeneration heralded a series of studies that provided evidence for a direct involvement of sleep-wake related neuronal mechanisms in progressive neuronal loss [19]. Finally, studies on nocturnal epilepsy and complex motor parasomnias have broadened our understanding of the neurobiology of consciousness and emotional behaviours [20].

5 The neurobiology of sleep and its relevance for the fields of neurology and psychiatry: significant developments in our understanding of the neurobiology of sleep have implications for our understanding of neurological and psychiatric disorders. First, neuroplasticity supporting memory consolidation and learning processes were shown to rely upon sleep and have major implications for both the healthy, the aging and the diseased brain [21,22]. Secondly, recent studies support Peron's traditional idea of a restorative function of sleep by removing neurotoxic substances accumulated in the brain during wakefulness [23]. Thus, getting adequate sleep may have a neuroprotective effect. Developing our knowledge of the neurobiology of sleep and its alterations requires new methodological developments, and the use of promising translational and animal approaches facilitating translation of findings from animal models to clinical situations.

Despite these important and exciting new insights, the opportunities of diagnosing and treating SWDs are frequently not adequately addressed or are neglected in neurological and psychiatric practice (apart from a few specialized research centres). In most countries, this is certainly in part the result of insufficient information about sleep during medical education. Likewise, sleep research and sleep medicine have been actively pursued in only a few neurological and psychiatric departments throughout Europe [1].

The present review results from a collaborative effort of a group of European experts in the fields of interest. It summarizes the most important recent research and medical findings in the field of sleep research and sleep medicine and discusses the promise they bear for the next decade. The results of these 
analyses form the basis of recommendations for priorities in education, medical practice and research in the fields of sleep, neurology and psychiatry.

\section{Methods}

This review summarizes the results of a symposium organized by the chair (CB) and co-chair (LFS) of the Alpine Sleep Summer School, one of the premier European forums for postgraduate education in sleep medicine. During this conference, held from 19 to 21 June 2014 in Baveno (Italy), 26 experts from the domains of sleep research, circadian research and sleep medicine including the last three presidents of the European Sleep Research Society (TP, CB, PP) and the president-elect of the World Association of Sleep Medicine (LFS) - met in 10 small workshops to discuss the major findings within these fields in the past decade, and their application to contemporary medicine. A standardized approach was used in all workshops and two main sessions started and completed the meeting. The two first authors worked on a draft paper based on the written summaries of material produced from these workshops. The initial contributors then revised the draft extensively. Mostly review papers are referenced here to allow the interested readers to deepen their knowledge of the topics of interest.

\section{Results (listed by workshop topic, from each of 10 workshops)}

\section{Sleep, memory/neuroplasticity and recovery from brain damage}

State of the art

Healthful and sufficient sleep is essential to optimal cognitive function in humans and animals, whilst disruptions of sleep is a hallmark of a wide range of neurological and mental disorders. In the past decade, significant advances have been accomplished especially in the field of memory research, from different perspectives spanning both synaptic and network levels [22]. Experience-dependent changes occur in synaptic assemblies during sleep in rodents [24], and many studies have shown that the recall of recently learned information is better after an episode of sleep than of wakefulness [25]. Models of sleep and memory consolidation have been developed and refined. One prominent approach focuses upon the idea that local neuronal assemblies activated during learning are 'depotentiated' during subsequent sleep, making room for new learning whilst creating an enduring memory trace (Fig. 3, left inside) [26]. Another prominent conceptualization, based on both animal [27] and human [28-30] studies, supports the hypothesis that sleep promotes reactivation and transfer of information for consolidated storage in distributed cortical networks (Fig. 3, right inside). Recent research has highlighted the importance of individual microstructural sleep features - e.g. slow oscillations, spindles, hippocampal ripples and ponto-geniculo-occipital waves - for memory-related processes [22,31] and attributed both unique and overlapping roles to these features.

\section{Future}

Notwithstanding this, our understanding of the functions of sleep in supporting memory and the integrity of other cognitive functions remains incomplete. Also, the effect of sleep as treatment target to promote neuroplasticity and functional recovery after brain damage is largely untested.

First, we need to better understand the genetic and epigenetic factors that explain substantial interindividual differences in the benefits of sleep on memory and cognition. Such knowledge is expected to lead to the discovery of novel targets for innovative
Figure 3 Neuroplasticity mechanisms supporting memory consolidation and learning processes during sleep are suggested by both animal and human studies. The mechanisms involved are still unclear. A first hypothesis is that local neuronal assemblies activated during learning are 'depotentiated' during subsequent sleep to make room for new learning whilst creating an enduring memory trace (upper left). A second hypothesis is that sleep promotes reactivation and transfer of information for consolidated storage in distributed cortical networks (upper right).

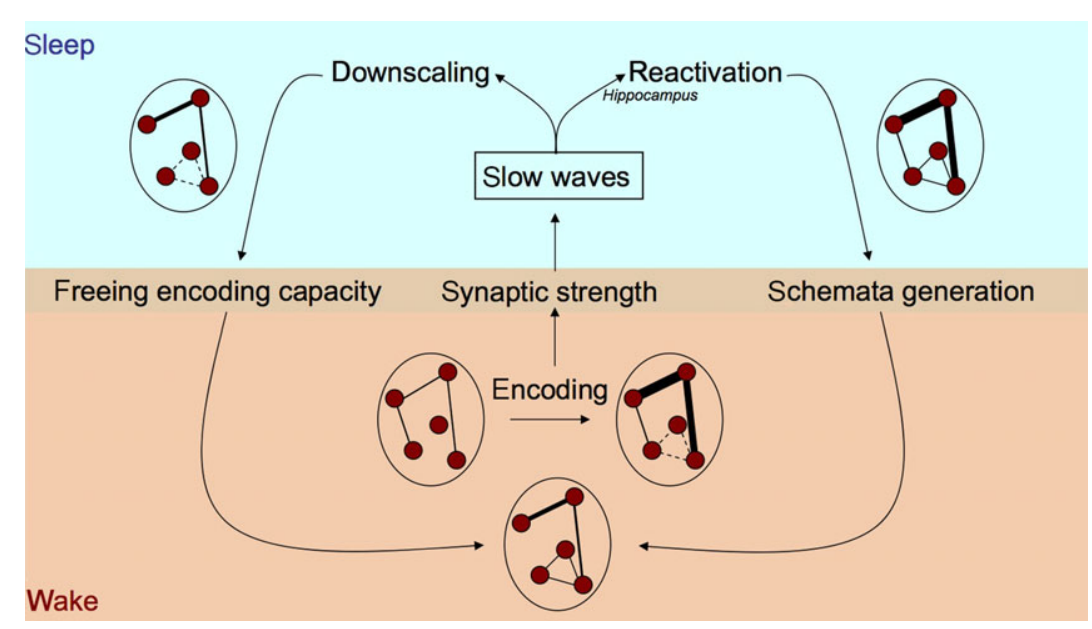


pharmacotherapy. There is also a need to more fully identify the cellular and molecular mechanisms sustaining the effect of sleep (and sleep deprivation/disturbances) on memory. A wide range of techniques, from intracellular and unit recordings to brain imaging, can be employed further to explore these mechanisms. Using interventionist approaches in animal models and non-invasive stimulation techniques in humans, manipulating distinct aspects of sleep should allow the establishment of causality chains in the cascade of neural events underlying the impact of sleep on memory.

Secondly, it is important to assess clinically the impact of sleep loss (e.g. secondary to shiftwork, chronic sleep restriction) and sleep disorders (e.g. SDB, insomnia/sleep fragmentation) upon memory and more generally upon cognitive functions.

Thirdly, experimental and clinical studies are needed to test whether pharmacological and non-pharmacological sleep-related interventions can be used to promote recovery from brain damage, which is known to rely on neuroplasticity processes and 'relearning' [32,33].

Key points

- Promoting adequate sleep is crucial to cognitive functioning and learning, especially in a society that values 'work around the clock'.

- Interventions to obtain adequate sleep are expected to promote neuroplasticity and cognitive processes during rehabilitation after brain damage and during cognitive behavioural therapies.

- A 24-h perspective is needed to fully understand neuroplasticity and cognitive processes in both health and disease, a perspective that is not restricted to an opposition between wakefulness and sleep but rather envisions cognition and memory/ learning as dynamic processes spanning the whole day-night cycle.

\section{Insomnia and mental health}

\section{State of the art}

Insomnia is defined by subjective complaints of prolonged sleep latency, difficulties to maintain sleep or early morning awakening, accompanied by negative daytime consequences. Chronic insomnia (symptom duration longer than 3 months) is the most prevalent sleep disorder, afflicting over $10 \%$ of the population. It contributes significantly to the risk or severity of cardiovascular, metabolic, mood and neurodegenerative disorders $[4,7,34]$. Insomnia with short sleep represents a subtype that is most at risk for adverse consequences [35].

The diagnosis of insomnia is based on subjective complaints. However, actual morbidity frequently varies widely from patient perception. Polysomnography (PSG) can be useful to rule out secondary forms of insomnia [such as SDB and RLS/periodic limb movements in sleep (PLMS)]. In addition, PSG (but also actigraphy) provides estimates of objective sleep and wake duration. Although meta-analyses indicate significant PSG deviations when averaged over a large sample of insomniacs [36], PSG measures do not deviate from normal in many individual cases and do not capture the subjective experience of being awake. As a consequence, subjective wakefulness appears to be a separate dimension that awaits appropriate quantification. Suggested quantitative electroencephalography (EEG) measures may be complemented in the future by methods (e.g. high density EEG, cortico-cortical connectivity etc.) reflecting neuronal correlates of consciousness in healthy people and patients with disorders of consciousness. Insomnia subtypes proposed in previous diagnostic classification systems (DSM, ICSD) were unsatisfactory and have been abandoned. Clearer subtypes may emerge from multivariate assessment that is less focused on sleep phenotypes only. Careful multivariate matching of good and poor sleepers to reduce heterogeneity has recently revealed neural correlates of resilient versus vulnerable sleep, including activation and integrity of brain white and grey matter [37]. The preferential involvement of specific areas of the brain in the pathophysiology of insomnia is further supported by observation of new onset insomnia in patients with focal brain damage (e.g. with left dorsomedial frontal lobe damage) [38].

Non-pharmacological treatment of insomnia has been improved by the development of cognitive behavioural approaches that were proved to be as effective as hypnotics [39]. Pharmacological treatment, traditionally related to benzodiazepines and so-called z-drugs, was recently enriched by the modulation of other arousal networks such as histaminergic and hypocretinergic neurons $[40,41]$. The role and advantages of these approaches still need to be understood.

\section{Future}

The next decade will show whether the dimensional view of research-domain-oriented criteria (RDoC) is more valuable than previous categorical diagnostic classifications [42]. It is arguable to interpret the presence of sleep oscillations as a change in the state of consciousness. Recent observations of the possible coexistence of regionally dissociated brain activity patterns during both behavioural sleep and wakefulness, with some brain areas being active whilst others show sleep signs, may offer a new perspective in the evaluation of insomnia. Neurophysiological research in the next decade may reveal the relative contribution of 
disturbed perception, duration and fragmentation of sleep to the adverse consequences of insomnia. Similarly, brain imaging holds a high promise to reveal subtypes, causes and consequences of insomnia.

Overall, the future data-driven definition of insomnia subtypes with differential underlying mechanisms also promises more personalized treatment. Although cognitive behavioural treatment seems to be more satisfactory than pharmacological treatment, it still leaves many patients symptomatic. Unfortunately, determination of polymorphisms or copy number variations to mechanistically understand the heritability of insomnia is hampered by the lack of specific insomnia phenotyping in the large aggregated cohorts of genotyped and brain-imaged individuals presently available. A challenge for the next decade is to reach these individuals to collect their lifetime history of sleep vulnerability [43]. The use of internet-based methods within a stepped care framework would allow this at minimal cost [44] and will help to evaluate whether early insomnia treatment is capable of preventing consequences like cardiovascular disease, depression/suicidality and cognitive dysfunction.

\section{Key points}

- Insomnia contributes to the risk or severity of cardiovascular, metabolic, mood and possibly also neurodegenerative disorders, making it highly relevant for the next decade to determine whether successful sleep interventions protect against these adverse consequences.

- Detailed endophenotyping, coupled with behavioural and environmental assessment in longitudinal cohort studies, holds significant promise towards elucidating the basis of these risks.

- New approaches are expected to improve the diagnostic accuracy (and the characterization of insomnia subtypes) and treatment of insomnia.

\section{Circadian biology, psychiatric and neurological disorders}

\section{State of the art}

A proper function of the circadian timing system is mandatory for normal sleep and optimal physiological function of the brain and the body, and arises from a clock 'machinery' which includes the retino-hypothalamic pathway, conducting environmental timing signals, and the suprachiasmatic nuclei, the mammalian 'master clock'. This 'master clock' synchronizes cellautonomous circadian clocks in nearly all cells of the brain and body, conveying circadian control upon all major neurotransmitter systems.
It is therefore not surprising that disturbances of circadian functions and a circadian variation of clinical manifestations are common in a variety of neuropsychiatric disorders such as depressive and affective disorders, stroke, Alzheimer's disease, parkinsonism, RLS and traumatic brain injury $[1,10]$. In affective disorders, prominent diurnal variations of mood have been well known for decades and recognized as predictors of the outcome of illness episodes. Onset of stroke appears to be more frequent in the morning hours. In RLS sensorimotor symptoms follow inversely the core temperature course. In Alzheimer's disease a correlation was found between fluctuations of cognitive functions and measures of circadian output, and later stages of disease are associated with profound shifts in the behavioural phase (so-called 'sundowning') [45]. Specific interventions targeting the circadian system, such as sleep deprivation and intensive light, were shown to improve symptomatology in mood disorders and Alzheimer's disease, and simple behavioural circadian reinforcement can improve mild dementia and well-being in elderly individuals [15].

Multiple mechanisms are involved in the above mentioned bidirectional interactions between circadian functions and neuropsychiatric disorders. Polymorphic variations of clock genes contribute to psychopathology in bipolar disorder, pointing to either a causative involvement of the central circadian clock [46] or its altered response to external 'zeitgebers' (e.g. light-dark cycle). Conversely, different behaviours (e.g. bad sleep hygiene) may also impact on clock gene expression [47], and circadian interventions have direct effects on neurotransmitter systems [46]. Furthermore, brain damage may disturb the clock machinery and lead to circadian disturbances [48]. Finally, drugs frequently used in treating neuropsychiatric disorders (e.g. lithium, valproic acid) have direct targets within the circadian machinery, and biomarkers from circadian pathways have been shown to be useful for diagnostic classification and development of new treatments in psychiatry [49]. However, circadian concepts and interventions have entered the clinical domain very little so far.

\section{Future}

One clear future goal is the consideration of molecular circadian parameters in psychiatric and neurological studies. For example, new in vitro methods such as the use of fibroblasts should be used to investigate circadian parameters and their relation to treatments and psychopathology [50]. Moreover, further studies on circadian clock genes in psychiatric and neurological patients are needed, since these genes generate a 
temporal pattern of reactivity of brain cells to various stimuli. To better treat these patients, large-scale multicentre controlled treatment studies manipulating/ depriving light/sleep are needed. Besides targeting typical patients with affective disorders, chronotherapeutic approaches (including light) should also be tested in neurological populations such as Alzheimer's patients and other brain disorders (e.g. parkinsonism) accompanied by severe circadian and SWDs.

Disrupted circadian timing (e.g. shift work) has a detrimental effect on the course and symptomatology of psychiatric and neurological disorders, prompts illness relapse, and should be closely monitored to improve outcomes in these disorders. The circadian clock itself should be studied as a potential psychopharmacological drug target. 'Time-of-day' needs to be regarded as an important factor and studied to evaluate psychopharmacological drug efficacy and its therapeutic window [51].

Finally, circadian clock parameters hold considerable promise for endophenotyping of psychiatric and neurological disorders. To further exploit this potential, studies should be performed classifying neuropsychiatric disorders based on phenotypic circadian dimensions. This characterization has been made possible on the one hand by new multichannel recording devices capable of ambulatory long-term measurements of motor activity, heart rate, temperature and sleep, and on the other hand by detailed molecular characterizations directly from patient cells.

Key points

- Methodological advances have made the study of circadian functions and phenotypic circadian dimensions possible not only in healthy subjects but also in patients.

- A better and wider implementation in clinical practice is needed and expected to have important implications for the diagnosis and treatment of neurological and psychiatric disorders.

\section{Sleep-wake disorders* and their impact on the diagnosis and outcome of neurological and psychiatric disorders}

State of the art

A normal sleep-wake cycle depends on the integrity of the brain, which explains the high frequency of SWDs in neurological and psychiatric patients (Table 1). Conversely, since sleep has fundamental restorative functions for the brain, sleep disorders

*other than circadian disorders (above), sleep apnoea (following) and RLS/PLMS. may influence the course and outcome of neurological and psychiatric disorders, and may even be directly and causally implicated in these diseases.

In the following areas, a better understanding of the link between sleep-wake function (SWF)/SWDs and neurological disorders has direct clinical implications.

First, SWDs can represent the main or first manifestation of a neurological or psychiatric disorder. RBD has been recognized to be the first symptom of such neurodegenerative diseases as Parkinson's disease, dementia with Lewy bodies and multisystem atrophy [52]. Insomnia, EDS/hypersomnia/fatigue can be the first or only manifestation of stroke and neurodegenerative and mood disorders [7,8,53]. Subtle disturbances of SWF and circadian function can be observed in preclinical Alzheimer's disease, whilst clinically evident disturbances are often seen in advanced dementia and parkinsonian syndromes [9]. It remains unclear whether treatment of these SWDs may prevent the subsequent development/evolution of the associated neurological and psychiatric disorders.

Secondly, detrimental effects of sleep loss and disturbance on neuronal function/integrity may include a diminished clearance of toxic substances such as amyloid out of the brain and contribute also to the proinflammatory state associated with these disorders [23].

Thirdly, in addition to its modulating effect on the frequency of migrainous and epileptic attacks, sleep disturbances and sleep loss seem to have an important role on stroke evolution and recovery in both animal models and patients [54].

Fourthly, the observation of insomnia, EDS and RBD in the context of multiple sclerosis, limbic encephalitis and paraneoplastic syndromes raises questions about the frequency and implications of autoimmune mechanisms in SWDs in general $[55,56]$.

These advances contrast with still unsolved diagnostic issues concerning SWDs in neurological and psychiatric populations.

(a) A variety of scales and diagnostic criteria have been developed for the diagnosis and rating of symptoms of RBD, RLS, PLMS, narcolepsy, sleep disorders in Parkinson's disease, sleep-related frontal lobe (hypermotor) epilepsy and parasomnias. Unfortunately only scarce data exist on their diagnostic accuracy or correlation with objective measures.

(b) Conventional sleep-wake assessment relies on criteria developed for healthy people. In patients with brain damage affecting the sleep-wake EEG mechanism, however, these criteria are not always appropriate $[56,57]$.

(c) The development of reliable portable systems has opened the possibility of measuring sleep outside the constraints of the sleep laboratory. 
(d) The impact of our knowledge on sleep medicine has not yet been incorporated into the clinical practice of neurology.

Advances in the treatment of SWDs in neurology were made in the field of narcolepsy, circadian disorders in dementia [15], RLS and nocturnal epilepsy. Conversely, evidence for treatment of SWDs in stroke, Parkinson's disease, head trauma and multiple sclerosis is still limited mostly to single cases or small series.

\section{Future}

Treatment of SWDs may have an impact on the evolution and outcome of neurological and psychiatric disorders. For this reason, symptoms of disordered sleep should be integrated routinely into the clinical history of neurological and psychiatric patients. Helping to achieve this goal, diagnostic criteria for sleep disorders in neurological and psychiatric patients should be developed and validated. Standard tests are often useful. However, improvements in portable recording systems are necessary.

\section{Key points}

- Identification of insomnia, RBD, EDS/hypersomnia/fatigue and other SWDs has diagnostic value for the early recognition of depression/mood disorders and neurodegenerative disorders such as Parkinson's and Alzheimer's disease.

- Early diagnosis of SWDs may offer a new 'therapeutic window' with a favourable impact on the evolution and outcome of such disorders as epilepsy, stroke, migraine, parkinsonism and Alzheimer's disease.

\section{Sleep disordered breathing, neuropsychiatric consequences and neurological/psychiatric disorders}

\section{State of the art}

Sleep-disordered breathing is of major clinical and socioeconomic relevance due to the high prevalence and the worse outcome of affected patients. SDB includes several phenotypes characterized by different degrees of upper airway obstruction [snoring, upper airway resistance syndrome and obstructive sleep apnoea (OSA)] or impairment of respiratory drive or minute ventilation [central sleep apnoea (CSA), Cheyne-Stokes respiration (CSR), opioid-induced sleep apnoea, hypoventilation syndromes].

Sleep-disordered breathing is associated with (i) EDS, fatigue or insomnia, (ii) neuropsychiatric symptoms and (iii) increased cardiovascular and cerebrovascular morbidity and mortality (e.g. representing an independent risk factor of stroke and affecting also its outcome $[2,13])$.
The different SDB phenotypes may present some but not all of these clinical features. Gender, genetic and environmental factors are amongst the many factors that are involved in determining the different phenotypes (and complications of SDB). Treatment of OSA with CPAP promotes a mild but significant reduction in blood pressure, especially in treatment-refractory hypertension and sufficiently CPAP-adherent patients, as well as improvement of EDS. It has been demonstrated that a moderate but sustained weight reduction can prevent the progression of OSA or even cure mild OSA in obese patients. Regardless of the many open questions on pathophysiology, clinical presentation, cardiovascular and metabolic consequences, the differentiation of the phenotypes and the differential therapy, the workshop focused on the influence of SDB on neurological and psychiatric implications.

About $5 \%-10 \%$ of CPAP-treated patients continue to complain of EDS despite normalization of ventilation during sleep: a decreased central cholinergic activity can play a role in the pathophysiology of residual EDS.

Most data on neurocognitive impairment and outcome focus on OSA whilst there is a lack of information on CSA and CSR. This points out the importance of an interdisciplinary approach, including other subspecialties, e.g. cardiology and nephrology.

Cross-sectional studies showed that OSA is associated with an impairment of memory function, attention and executive functions. A mild cognitive impairment and dementia were found in older women with OSA, whilst data on other groups are lacking [11]. Recent studies showed progressive structural changes in cortical and subcortical brain areas, including the putamen and hippocampus [58,59], associated with impairments in cognition, mood and sleepiness [60]. The morphological changes develop from acute swelling to chronic loss of volume in untreated OSA patients. Hypoxia and changes between hypoxia and reoxygenation rather than sleep fragmentation substantially influence brain structural changes. Structural brain changes are potentially reversible by ventilatory therapy [60]. In addition, treatment with CPAP objectively improves attention [61].

Anxiety and depression are frequent comorbidities in OSA and may influence the symptomatology and the adherence to CPAP therapy [62]. They may also play a role in the pathogenesis of OSA, because of the direct influence of drug therapy and increased body weight.

\section{Future}

In general, causes and consequences of residual EDS and neurocognitive impairments despite efficient 
CPAP therapy require better evaluation. Psychological assessment for detection of anxiety or depressive disturbances should be included in the clinical guidelines for the evaluation and follow-up of patients with OSA. Furthermore, additional longitudinal studies should be performed in treated and untreated OSA patients in order to define the risk of developing mild cognitive impairment and dementia.

Prospective trials are also needed to assess more precisely the role of OSA, CSA and CSR (and their treatment) as risk factors for stroke and as modulatory of its outcome.

Treatment alternatives to CPAP should be better evaluated especially in terms of their impact on neurological and psychiatric symptoms, including oxygen, positional therapy, mandibular advancement and hypoglossus stimulation. Pharmacological treatment may be developed for specific manifestations of SDB (e.g. upper airway muscle function, loop gain etc.) and may help in reducing the overall burden of the disease.

Key points

- Prospective clinical and neuroimaging studies highlight the importance of early diagnosis and successful treatment of OSA to prevent EDS, neuropsychiatric/cognitive symptoms and stroke.

- The relationship between SDB/OSA and dementia/ neurodegenerative disorders remains controversial.

- Treatment alternatives to CPAP should be developed and/or better evaluated.

\section{Narcolepsy and central (neurogenic) hypersomnias}

State of the art

Central hypersomnias are disorders characterized by EDS and/or increased sleep (hypersomnia) that are not due to disturbed nocturnal sleep or misaligned circadian rhythm [63]. Central hypersomnias include narcolepsy, recurrent hypersomnias (Kleine-Levin syndrome, catamenial hypersomnia), idiopathic hypersomnia, behaviourally induced insufficient sleep syndrome (which is not strictly a brain disorder but rather a lifestyle consequence) and hypersomnia secondary to medical, substance-related, neurological or psychiatric disorders.

In recent years, major advances were achieved in the field of narcolepsy with cataplexy (narcolepsy type 1). Pathophysiologically, the discovery that deficient hypocretin neurotransmission causes narcolepsy led, also thanks to new animal models, to a better understanding of the neurochemistry and neurophysiology of narcolepsy as a hypothalamic disorder [64]. Expanding knowledge about the hypocretin system also improved our understanding of sleep-wake regulation in general and of the interplay of sleep, the autonomic system, metabolism, body temperature regulation, metabolism, motor regulation, emotional control, addictive behaviour and cognition [65]. Related research suggested a role of impaired hypocretin neurotransmission in other neuropsychiatric disorders such as head trauma, Parkinson's disease and Alzheimer's disease and improvement of insomnia following antagonization of the hypocretin system $[19,41,66,67]$.

Mechanisms of hypocretin cell loss in human narcolepsy remain unclear. Although indirect evidence for an autoimmune mechanism is accumulating, a final proof is still lacking. Narcolepsy appears to arise from a genetic predisposition and a variety of, possibly cumulating, environmental factors including infections (e.g. streptococcus, influenza virus), vaccinations (e.g. H1N1) and others (e.g. head trauma) [68]. The involvement of other neuronal systems remains possible.

Clinically a distinct childhood phenotype, an association with obesity, vigilance and cognitive disturbances, and frequent comorbid anxiety and depression were only recently characterized [69]. Conversely, reliable criteria for the diagnosis of the borderland of narcolepsy that is narcolepsy without cataplexy, idiopathic hypersomnia and behaviourally induced insufficient sleep syndrome, hypersomnia associated/ secondary to medical disorders, substance-related hypersomnia and hypersomnia related to neurological or psychiatric disorders are still lacking.

Effective treatment includes lifestyle changes, antidepressants, as well as stimulants and sodium oxybate (reaching class A evidence for the latter two).

\section{Future}

A better understanding of the borderland of narcolepsy is important, including the role of chronic sleep deprivation and psychiatric comorbidities as co-determinants of central hypersomnias.

Narcolepsy research is expected to identify (i) the (autoimmune?) mechanism of presumed hypocretin cell death and (ii) the exact role of the hypocretin system (and possibly other neurotransmitter deficiencies) in the pathophysiology of narcolepsy but also in sleep-wake regulation and such other physiological functions as reward, motivated behaviours and metabolism.

From a diagnostic point of view, better screening tools, new tests to assess sleepiness, disturbed vigilance, cataplexy and their severity in narcolepsy are needed.

Concerning treatment, new generation stimulants such as pitolisant (an agent promoting histaminergic neurotransmission) and JZP-110 (an agent promoting both noradrenergic and dopaminergic neurotransmis- 
sion) are under investigation and may become available soon [70]. Efforts are currently devoted also to the development of appropriate hypocretin agonists.

\section{Key points}

- The borderland of narcolepsy should be better characterized, eventually improving our understanding of psychiatric and behavioural co-determinants of hypersomnia.

- A better understanding the aetio-pathophysiology of narcolepsy will expand our knowledge on the role of hypocretin in sleep-wake and other central nervous system functions, but also on neuroimmunological brain disorders.

\section{Restless legs syndrome (Willis-Ekbom disease) and periodic limb movements in sleep}

\section{State of the art}

Although RLS is one of the most prevalent neurological disorders it is still under-diagnosed, particularly in infancy or in conditions such as pregnancy, anaemia or renal failure $[1,71]$. The frequency in neurological and psychiatric patients is particularly high [72]. No biological diagnostic markers are available for RLS and the current diagnostic criteria lack specific thresholds for symptom severity, frequency or impact on quality of life.

The exact pathophysiology of RLS, for which no reliable animal model is established, is still unknown. The rapid efficacy of low doses of dopamine agonists (DAs), the circadian oscillation of endogenous dopamine, the worsening of RLS by dopamine antagonists and a multitude of neurophysiological data suggest a primary spinal cord involvement arising from a dysfunction of the dopaminergic hypothalamo-spinal inhibitory descending pathway [73]. This may in turn be related to a deficient central nervous system iron transport/storage [74]. Strong evidence also supports a role of genetic factors [75]. In particular, early-onset RLS is highly familial and a positive parental history for RLS is found in more than $70 \%$ of children [76]. Nevertheless, neither familiar linkage analysis nor genome-wide association studies have been able to identify specific gene mutations or polymorphisms responsible for the disease, suggesting that RLS is a complex polygenic phenotype.

Approximately $70 \%-90 \%$ of adult RLS subjects present with PLMS. Such movements allow high diagnostic sensitivity for RLS, but only low specificity. Their causative role in inducing sleep instability, their relation to sleep breathing disorders and their cardiovascular long-term consequences are still debated [5].
In the short term, suppression of PLMS does not affect cortical arousals, and scientific evidence to warrant treatment of PLMS is still lacking [77]. The pathological significance of PLMS without RLS, often incidentally found in PSG studies, is even more uncertain.

Several diseases have symptoms that are often confused with those of RLS, and other neurological and psychiatric conditions are associated with higher rates of RLS, including migraine, multiple sclerosis, spinal cord lesions, parkinsonism and mood disorders.

Chronic and clinically significant RLS can be treated with either a non-ergot DA or an $\alpha-2-\delta$ calcium channel ligand [78]. DAs might be a more appropriate choice in the presence of depression and overweight. Since $\alpha-2-\delta$ ligands can alleviate chronic pain and may be helpful in treating anxiety and insomnia, the presence of any of these comorbidities may favour their use. In refractory RLS, oral prolonged release oxycodone-naloxone should be considered [79].

The most relevant clinical problem in the management of RLS is represented by the phenomenon of augmentation, an unexplained increase of RLS symptom severity occurring during long-term therapy with dopaminergic agents. No evidence-based guidelines on the management of augmentation are available.

\section{Future}

Future clinical studies should address (i) the impact of severity and frequency of symptoms on the impact of RLS (e.g. quality of life), (ii) the characterization and relevance of different RLS subtypes (e.g. primary versus secondary RLS, familial versus non-familial RLS, RLS with and without comorbidities).

Therapeutically, DAs provide effective treatment for RLS/PLMS but less for sleep instability, whilst $\alpha-2-\delta$ calcium channel ligands show the opposite effects. Therefore, future studies should consider possible combined therapy or new compounds in RLS treatment and clarify the therapeutic role of iron supplementation in RLS patients with both normal and deficient ferritin levels. Treatment for many RLS subpopulations or comorbidities such as uraemia, pregnancy and depression remains unclear/ unproven. Finally, long-term prospective, interventional studies will evaluate the effects of RLS/PLMS treatment on sleep disruption and cardiovascular consequences.

Key points

- Restless legs syndrome is frequent, particularly in patients with neurological and psychiatric disorders. 
- The consequences (e.g. cardiovascular complications) of different forms of RLS are unclear.

- New approaches are needed for a better diagnostic accuracy (e.g. primary versus secondary forms) and for long-term treatment of idiopathic and secondary RLS.

\section{Sleepwalking, REM sleep behavior disorders, sleep-related hypermotor epilepsy and the concept of state dissociation}

State of the art

Complex parasomnias such as sleepwalking, RBD and sleep-related hypermotor seizures (SHS) are disorders that feature sleep-related complex motor behaviour that can even lead to self-injury and violence against others, representing also a challenge in forensic medicine [80]. The differentiation between complex parasomnias and SHS has implications for treatment but may be clinically difficult. Pathophysiologically, a 'state dissociation' - defined as the abnormal mixture of elements from different states of being (wakefulness, non-rapid-eye-movement sleep and REM sleep) - was proposed to explain these disorders [81]. Genetics, alcohol, psychosocial factors, medications and brain damage (e.g. traumatic brain injury) are thought to be involved in causing such a state dissociation.

Whilst the concept of state dissociation is clinically helpful, it lacks clarity regarding the exact underlying mechanisms and thus does not allow objective or quantitative evaluation and differentiation of complex parasomnias from sleep-related hypermotor epilepsies. In recent years, it has been shown indeed that sleep is also locally regulated [82,83]. This observation, together with single-photon emission computed tomography and neurophysiological data documenting the coexistence of selectively activated brain areas during sleep, gave support to the concept of state dissociation $[84,85]$.

One way to unravel the intricate relationship between local ('smaller scale') and global ('larger scale') patterns of brain activity and how they are associated with distinct behaviours has been provided by the continuous improvement of techniques for recording brain activity with very high spatial resolution. For example, the introduction of high density surface EEG highlighted local differences of cortical activity during wake and sleep. Moreover, intra-cerebral recordings in animals and humans provided evidence of a rather continual transition between wakefulness and sleep with the possibility of the normal coexistence of elements of both states $[83,86]$.
Thus, these investigations prove that transitions between global brain states are not spatially and temporally uniform but show a high degree of topographic variability - not only under pathological but also under physiological conditions.

\section{Future}

Clinically, quantification of the mixture of elements of wakefulness and sleep is necessary as attempted already by some authors [87].

This may be achieved by the application of powerful concepts from network analysis such as segregation and integration [88]. Segregation and integration are 'universal' concepts in the sense that they are applicable to any complex system, i.e. systems consisting of large numbers of interacting parts such as the brain.

A better assessment of the different elements configuring the dissociated states may eventually improve our ability to monitor and evaluate transitions between physiological and pathological brain dynamics (e.g. in patients awakening from coma or at the transition from vegetative state to minimally conscious state [89]), and possibly also contribute to a better prediction of responses to specific treatments. Furthermore, it may also elucidate other neuropsychiatric or behavioural disorders that may emerge from a disturbed balance of segregation and integration within central neuronal networks. Finally, investigating disorders such as sleep-related focal epilepsy, both genetically and structurally, may give insights into understanding the relationship between genes, epileptogenesis and arousal regulatory processes [90].

Key points

- State dissociation is a useful concept to describe complex parasomnias and SHS but appropriate measures of it are still lacking.

- The development of quantitative measures of dissociation will be of help not only in sleep medicine but also more generally for the understanding and management of disorders of consciousness such as coma and minimally conscious state.

\section{New methodological approaches}

State of the art

The clinical evaluation of sleep has traditionally relied on qualitative analysis of EEG recordings. During the last decade, however, quantitative EEG analyses and other recording techniques have revealed exciting new insights into sleep mechanisms. Qualitative criteria for visual scoring of sleep stages and events were 
introduced in the 'paper era' of sleep medicine and continue to be the basis of current rules [91]. Given the promise of quantitative EEG analyses, it is recommended for the future to establish an international consensus on data-driven standards for the scoring of sleep for clinical purposes.

During the last decade, sleep research in humans and animals has witnessed a dramatic increase in the number of electrodes used to record EEG (socalled high density EEG), as well as complementary use of magnetic resonance imaging, magneto-encephalography and near infrared spectroscopy. The advanced recording approaches have been combined with in-depth signal analyses, including advanced multivariate analysis techniques (e.g. independent component analysis), source reconstruction and multivariate pattern classification to provide better insights about the spatiotemporal characteristics of sleep, including its underlying neuronal networks and their oscillations in spindles and slow wave frequencies [92-94]. Methods to evaluate the role of sleep in brain function and its response to stimulation have refined the use of traditional sensory stimulation and applied newer methods that bypass the senses and directly stimulate transcranially, by means of magnetic pulses and alternating or direct electric currents $[95,96]$.

Human studies have been complemented by breakthrough findings on the importance of sleep for brain function in animal studies using two-photon imaging $[23,24]$ and optogenetics [97,98], as well as by cellular and molecular studies exploring the roles of individual genes and signalling pathways contributing to the EEG features of sleep. In particular, transcriptomic characterizations have permitted the elucidation of cellular pathways important for the regulation of sleep onset and/or maintenance, whilst genetic studies have suggested the existence of specific ion channels or metabolic pathways that might alter sleep-specific brain oscillations. At the same time, multimodal imaging and multiunit multisite recordings have allowed detailed study of changes in brain connectivity or metabolite clearance at local and global levels in both humans and animals.

\section{Future}

Advanced methods like single cell calcium imaging and intracellular recording will further reveal the circuits and functions of sleep. Interventional approaches, including optogenetics and cell-specific pharmacology, bear the ultimate promise of applicability to improve sleep and its supportive role in brain function and cognition in humans. In addition, there are a number of challenges and opportunities to address.
A first opportunity relates to the development of an international consortium that assembles genome-wide association and functional imaging studies. Thus, genomic, electrophysiological and functional imaging databases should be consolidated and expanded together with internet-based survey of lifetime sleep habits, complaints and vulnerabilities. Moreover, collection of primary cells from patients and possibly trans-differentiation of these cells to neurons could provide unprecedented and easily accessible molecular information pertaining to behaviour, sleep and disease [99].

A second opportunity should promote the use of self-quantification of behaviour, environment and physiology by means of mobile and highly functional miniature devices, such as multiple microsensors. It is expected that massive databases will emerge from these new opportunities and will unravel important evaluations about sleep in natural environments and quantification of new sleep phenotypes. Moreover the increasing use of mobile communication devices will also support treatment of sleep disorders. Internetbased cognitive behavioural therapies for insomnia, as well as CPAP titration by means of home monitoring, have already been shown to be effective.

Key points

- A new international consensus on data-driven clinical scoring of sleep is needed. In a research setting, the picture of both healthy and pathological sleep can be further enhanced via new multimodal imaging/recording technologies.

- A high priority should be placed upon the integration of sleep considerations into large consortium data sets. This will require the continued close cooperation of sleep scientists and clinicians with experts in other disciplines, including information technologists, engineers and mathematical modellers.

- Molecular and cellular measures, when integrated with such information, should provide unparalleled opportunities for discovery of clinically relevant mechanisms regulating sleep.

\section{Translational and animal approaches}

\section{State of the art}

Several concepts have dominated the sleep field for decades. It was thought that (i) sleep slow waves are a global phenomenon with an intensity only determined by the need for sleep; (ii) sleep-wake cycles are under the control of a reciprocal inhibitory balance between GABAergic sleep-promoting nuclei located in the preoptic area and multiple arousal-promoting nuclei; and 
(iii) REM sleep is generated by reciprocal interaction between monoaminergic and cholinergic neurons located in the brainstem.

Studies in animals over the last decade have deeply modified some of these concepts. First, it is now clear that sleep is not a uniform global process and that its depth varies amongst cortical structures depending on their activity during previous waking. Further, slow waves characteristic of sleep can occur locally during prolonged wakefulness. In addition, recent studies suggested that sleep facilitates clearance of metabolites and toxins such as $\beta$-amyloid from the brain [23]. It was also shown that adenosine in astrocytes plays a role in sleep homeostasis.

At the system level, it has been shown that glutamate and GABA-glycinergic pathways control REM sleep and are responsible for muscle atonia. This is highly relevant to RBD and the mechanisms of cataplexy, suggesting that glutamate release from hypocretin/orexin cells, for instance, participates in signal transduction together with hypocretin/orexin peptides. Optogenetics was used to confirm that neurons located in the lateral hypothalamus, in particular those containing melanin-concentrating hormone and GABA, facilitate the onset and maintenance of REM sleep state [98]. Finally, animal models have been developed for a few sleep disorders including narcolepsy, insomnia/sleep deprivation, SDB (intermittent hypoxia) and RBD, and promising new models are under development for RLS/PLMs [18]. More generally, 'rest' in simpler organisms such as fruit flies and worms have proven powerful models for research, and conclusions about sleep-relevant genes and pathways elucidated in these 'simple' organisms starts to be validated in mice and human.

\section{Future}

A better phenotype characterization of sleep-wake in patients is now required to promote translational findings from animal models to clinical situations, and backwards. Besides the identification of sleep-wake neuronal populations, it is time to determine the mechanisms responsible for their functional connectivity and dynamics in basal conditions and under cognitive or physiological modifications. For example, the mechanisms responsible for inducing slow waves both globally and locally, as well as their relevant functions, remain to be identified. To fulfil these tasks, new methods to record and analyse brain network activity from cortical and subcortical structures with high temporal and spatial resolution (ideally cellular and molecular) should be developed (functional, structural, behaviour, longitudinal, magnetic resonance spectroscopy combined with high density EEG, multi- site recordings, and activity imaging at cellular level) both in animals and possibly humans. In addition, the development and improvement of genetic engineering in animal models for SWDs is needed in mice and other mammalian models using new methods such as clustered regularly interspaced short palindromic repeats (CRISPR). The use of optogenetics and pharmaco-genetics will also bring further answers, provided that biological markers allowing the specific study of subtypes of GABA and glutamatergic neurons are adequately identified, e.g. by using gene expression analysis. Finally, utilization of cellular models for individual features of sleep-wake state could permit molecular discoveries at small-scale resolution.

Key points

- Increased knowledge of basic sleep-wake mechanisms using upcoming methods such as optogenetics, calcium imaging and CRISPR is needed.

- The development of relevant animal models for SWDs should be pursued to empower real opportunities for translational research approaches.

\section{Discussion}

The aim of the workshop resulting in this paper was to group specialists representing the multidisciplinarity of both sleep medicine and science in order to summarize recent advances and discuss future perspectives related to the fields of neurology and psychiatry. The broad spectrum of knowledge and approaches in this workshop represented a true challenge, and led us to focus on the 10 topics discussed in this paper. Within each topic, specific research priorities were identified, which we have outlined above. However, connecting these 10 topics, several consensus conclusions and clinical priorities repeatedly emerge.

\section{State of the art and future perspectives}

Overlapping brain mechanisms regulating sleep-wake, psychiatric and neurological functions probably explain the high frequency of SWDs in neurology and psychiatry. However, SWDs are often under-diagnosed in both specialties. With respect to sleep medicine, a clear-cut separation between neurology and psychiatry appears particularly difficult and often unjustified. Patients with SWDs often require multidisciplinary management, and well exemplify the need for a holistic approach in medicine.

Our understanding of the pathophysiology of SWDs in neurology and psychiatry has greatly improved. Such SWDs as narcolepsy and RBD represent experiments of nature, the study of which led to a better understanding 
of brain mechanisms underlying not only sleep and wake regulation but more generally cognitive, motor, emotion and autonomic pathways. In reverse, however, the translation of basic sleep-wake research to clinical practice remains inadequate. The necessity of a bidirectional interaction between clinical/human and basic/animal approaches is challenged by the increasing separation between these fields of research, more and more specialized. Such a difficulty of transfer of knowledge is particularly well recognizable in chronobiology, where great advances in our understanding of physiological mechanisms have had only a limited impact upon the management of psychiatric and neurological patients so far.

Sleep seems to play a fundamental role in maintaining brain and mental health. Accordingly, an increasing body of data suggests a negative influence of such SWDs as insomnia, SDB and circadian disturbances on the incidence, evolution and outcome of several neurological and psychiatric disorders. Detrimental interactions have also been documented for SWDs and learning/cognitive abilities in children, cerebrovascular disorders in adults, and neurodegenerative disorders in the elderly population. Systematic studies, including treatment interventions, are needed to prove causal relationships between most SWDs and neurological/psychiatric disorders.

With regard to research methodology, several new techniques such as functional and structural neuroimaging, high density EEG and intracranial recordings in patients, as well as optogenetics, calcium imaging and recombinatorial genetic engineering technologies in animals, are revolutionizing assessments of SWF and disturbances in animal and humans. Circadian rhythms are maintained by a set of classic clock genes that form complex and self-regulatory loops [100]. Recent genome-wide association studies have identified some loci that may contribute to the regulation of sleep duration $[101,102]$. These advances contrast with the limited standardization and validation in the diagnostic work-up of neurological and psychiatric patients with SWDs, which urgently needs to be improved.

Our improved understanding of the neurobiology of SWFs has led recently to the development of new pharmacological targets for the treatment of such SWDs as insomnia and narcolepsy. Pharmacology together with multiple ventilatory options for SDB, melatonin/light for circadian disorders and non-pharmacological approaches for insomnia altogether constitute the large armamentarium of treatment interventions available for SWDs in neurological and psychiatric patients. Unfortunately, few of these approaches have been tested and validated in largescale studies. Also, their impact on the long-term outcome of neurological and psychiatric disorders is often poorly known.

\section{Actions needed}

A discrepancy is clearly evident between the tremendous opportunity offered by our knowledge of SWFs and SWDs and its very limited application in the practice of neurology and psychiatry. Our working group identified four potential areas of action that could lead to a reduction of this gap.

1 Pre-graduate and postgraduate teaching in sleep and sleep medicine must be strengthened.

Teaching of sleep and sleep medicine is limited to a few hours in most medical curricula and typically a vertical integration between preclinical and clinical years is missing. Furthermore, most postgraduate training programmes in neurology and psychiatry only marginally include the management and implications of SWDs, or do not cover them at all. In Switzerland, for example, sleep medicine training will officially be included only in 2015 in the postgraduate requirements set by the national neurological society. More generally, awareness campaigns are also important not only for the general population but also for nonspecialized medical professionals, so that they become aware of the far-reaching implications of sleep health for patient well-being also in association with neurological and psychiatric symptoms and disorders.

2 The creation of academic, multidisciplinary sleep-wake centres should be fostered.

In such initiatives, medical, psychological and biological faculties should ideally be involved. The coexistence of both preclinical and clinical departments would favour not only the transfer of knowledge and a multidisciplinary approach in SWDs (see above) but also translational research projects including animal and basic science experiments.

3 Diagnostic approaches of SWDs in neurological and psychiatric patients must be simplified and validated.

There is an increasing demand for developing simple but reliable instruments to monitor SWFs and to diagnose SWDs in a natural environment and in a way that is more accessible and less costly than standard tests.

4 Treatment strategies for SWDs in neurology and psychiatry should be systematically tested (and supported).

It is important to move from cohorts composed of patients admitted to sleep centres to larger sample sizes drawn from the general population. The early diagnosis, treatment and whenever possible prevention of SWDs in neurology and psychiatry could yield great benefits in management of traditional neurological and 
psychological disorders, also in view of the increasing age of our society.

\section{Acknowledgements}

We thank Mrs Yasmin Belloni of the Neurocentre of Southern Switzerland and Fondazione Neuroscienze Ticino (FNT) for her support in the organization of the meeting in Baveno. This meeting was supported by the following non-profit organizations/foundations: Alpine Sleep Summer School (ASSS, www.sleep-summerschool.ch), FNT (www.fondazioneneuroscienze.ch), BENESCO (Bern Network for Sleep, Epilepsy and Consciousness), Fondazione Neureca (www.neureca.org), Sleep Neuroscience Association (SNA). Endorsed by: European Sleep Research Society (ESRS) and by the European Academy of Neurology (EAN).

\section{Disclosure of conflicts of interest}

C. L. Bassetti: international UCB narcolepsy advisory board, international JAZZ advisory board, consultation fees/speaker honoraria for Servier, Vifor, Zambon. C. Cajochen: speaker honoraria by Servier, Advisis AG and Takeda. R. Khatami: international UCB narcolepsy advisory board. G. J. Lammers: international UCB narcolepsy advisory board. C. Nissen: speaker honoraria of Servier. D. Riemann: consultation fee for ABBVIE 2013. M. Tafti: international UCB narcolepsy advisory board. The remaining authors declare no financial or other conflicts of interest.

\section{References}

1. Bassetti CL, Peigneux P, Dogas Z. ESRS European Sleep Medicine Textbook. Regensburg: European Sleep Research Society (ESRS), 2014.

2. Loke YK, Brown JW, Kwok CS, et al. Association of obstructive sleep apnea with risk of serious cardiovascular events: a systematic review and meta-analysis. Circ Cardiovasc Qual Outcomes 2012; 1: 720-728.

3. Young T, Finn L, Peppard O, et al. Sleep disordered breathing and mortality: eighteen-year follow-up of the Wisconsin Sleep Cohort. Sleep 2008; 31: 10711078.

4. Sofi F, Cesari F, Casini A, et al. Insomnia and risk of cardiovascular disease: a meta-analysis. Eur J Prev Cardiol 2014; 21: 57-64.

5. Ferini-Strambi L, Walters AS, Sica D. The relationship among restless legs syndrome (Willis-Ekbom Disease), hypertension, cardiovascular disease, and cerebrovascular disease. J Neurol 2014; 261: 1051-1068.

6. Iranzo A, Fernandez-Arcos A, Tolosa E, et al. Neurodegenerative disorder risk in idiopathic REM sleep behavior disorder: study in 174 patients. PLoS One 2014; 9: e89741.

7. Baglioni $\mathrm{C}$, Battagliese $\mathrm{G}$, Feige $\mathrm{B}$, et al. Insomnia as a predictor of depression: a meta-analytic evaluation of longitudinal epidemiological studies. J Affect Disord 2011; 135: 10-19.

8. Abbott RD, Ross GW, White LR, et al. Excessive daytime sleepiness and subsequent development of Parkinson disease. Neurology 2005; 65: 1442-1446.

9. Ju YES, McLeland JS, Toedebusch CD, et al. Sleep quality and preclinical Alzheimer disease. JAMA 2013; 70: $587-593$.

10. Wirz-Justice A. Chronobiology and psychiatry. Sleep Med Rev 2007; 11: 423-427.

11. Yaffe K, Laffan AM, Harrison SL, et al. Sleep-disordered breathing, hypoxia, and risk of mild cognitive impairment and dementia in older women. JAMA 2011; 306: 613-619.

12. Bassetti CL, Hermann D. Sleep and stroke. Handb Clin Neurol 2011; 99: 1051-1072.

13. Birkbak J, Clark AJ, Rod NH. The effect of sleep disordered breathing on the outcome of stroke and transient ischemic attack: a systematic review. $J$ Clin Sleep Med 2014; 10: 103-108.

14. Malow BA, Foldvary-Schaefer N, Vaughn BV, et al. Treating obstructive sleep apnea in adults with epilepsy. Neurology 2008; 71: 572-577.

15. Riemersma-van der Lek R, Swaab DF, Twisk J, et al. Effect of bright light and melatonin on cognitive and noncognitive function in elderly residents of group care facilities. A randomized controlled trial. JAMA 2008; 299: 2642-2655.

16. Lazar RM, Fitzsimmons BF, Marshall RS, et al. Remergence of stroke deficits with midazolam challenge. Stroke 2003; 33: 283-285.

17. De Cock VC, Vidhailet M, Arnulf I. Sleep disturbances in patients with parkinsonism. Nat Clin Pract 2008; 4: 254-266.

18. Toth LA. Animal models of sleep disorders. Comp Med 2013; 63: 91-93.

19. Kang JE, Limm MM, Holtzmann DM, et al. Amyloid(beta) dynamics are regulated by orexin and the sleep-wake cycle. Science 2009; 326: 1005-1007.

20. Tassinari CA, Rubboli G, Gardella E, et al. Central pattern generators for a common semiology in frontolimbic seizures and in parasomnias. A neurothologic approach. Neurol Sci 2005; 26: 225-232.

21. Mander BA, Rao V, Walker MP, et al. Prefrontal atrophy, disrupted NREM slow waves and impaired hippocampal-dependent memory in aging. Nat Neurosci 2013; 16: 357-364.

22. Rasch B, Born J. About sleep's role in memory. Physiol Rev 2013; 93: 688-766.

23. Xie L, Kang H, Xu Q, et al. Sleep drives metabolite clearance from the adult brain. Science 2013; 342: 373-377.

24. Yang G, Lai CS, Cichon M, et al. Sleep promotes branch-specific formation of dendritic spines after learning. Science 2014; 344: 1173-1178.

25. Stickgold R. Parsing the role of sleep in memory processing. Curr Opin Neurobiol 2013; 23: 847-853.

26. Tononi G, Cirelli C. Sleep and the price of plasticity: from synaptic and cellular homeostasis to memory consolidation and integration. Neuron 2014; 81: 12-34.

27. Wilson MA, McNaughton BL. Reactivation of hippocampal ensemble memories during sleep. Science 1994; 265: $676-679$.

28. Huber R, Ghilardi MF, Massimini M, et al. Local sleep and learning. Nature 2004; 430: 78-81. 
29. Maquet P, Laureys S, Peigneux P, et al. Experiencedependent changes in cerebral activation during human REM sleep. Nat Neurosci 2000; 3: 831-836.

30. Gais S, Albouy G, Boly M, et al. Sleep transforms the cerebral trace of declarative memories. Proc Natl Acad Sci USA 2007; 104: 188778-188783.

31. Nir Y, Staba RJ, Andrillon T, et al. Regional slow waves and spindles in human sleep. Neuron 2011; 70: 153-169.

32. Gorgoni M, D'Atri A, Lauri G, et al. Is sleep essential for neural plasticity in humans, and how does it affect motor and cognitive recovery? Neural Plast 2013; 2013: 103949.

33. Hodor O, Palchykova S, Baracchi F, et al. Baclofen facilitates sleep, neuroplasticity, and recovery after stroke in rats. Ann Clin Transl Neurol 2014; 1: 765777.

34. Yaffe K, Falvey CM, Hoan T. Connections between sleep and cognition in older adults. Lancet Neurol 2014; 10: 1017-1028.

35. Vgontzas AN, Fernandez-Mendoza J, Liao D, et al. Insomnia with objective short sleep duration: the most biologically severe phenotype of the disorder. Sleep Med Rev 2013; 17: 241-254.

36. Baglioni C, Regen W, Teghen A, et al. Sleep changes in the disorder of insomnia: a meta-analysis of polysomnographic studies. Sleep Med Rev 2014; 18: 195213

37. Stoffers D, Altena E, van der Werf YD, et al. The caudate: a key node in the neuronal network imbalance of insomnia? Brain 2014; 137: 610-620.

38. Koenigs M, Holliday J, Solono J, et al. Left dorsomedial frontal brain damage is associated with insomnia. J Neurosci 2010; 30: 16041-16043.

39. Riemann D, Perlis ML. The treatments of chronic insomnia: a review of benzodiazepine receptor agonists and psychological and behavioral therapies. Sleep Med Rev 2009; 13: 205-214.

40. Krystal AD, Richelson E, Roth T. Review of the histamine system and the clinical effects of $\mathrm{H} 1$ antagonists: basis for a new model for understanding the effects of insomnia medications. Sleep Med Rev 2013; 17: 263272.

41. Michelson D, Snyder E, Paradis E, et al. Safety and efficacy of suvorexant during 1-year treatment of insomnia with subsequent abrupt treatment discontinuation: a phase 3 randomized, double-blind, placebocontrolled trial. Lancet Neurol 2014; 13: 461-471.

42. NIH. RDoC: http://www.nimh.nih.gov/research-priorities/rdoc/index.shtml (accessed 11/05/2015). Research domain oriented criteria (RDoC).

43. Drake CL, Friedmann NP, Wright KP, et al. Sleep reactivity and insomnia: genetic and environmental influences. Sleep 2011; 34: 1179-1188.

44. Espie CA. 'Stepped care': a health technology solution for delivering cognitive behavioral therapy as a first line insomnia treatment. Sleep 2009; 32: 1549-1558.

45. Volicer L, Harper DG, Manning BC, et al. Sundowning and circadian rhythms in Alzheimer's disease. Am J Psychiatry 2001; 158: 704-711.

46. Benedetti F, Smeraldi E. Neuroimaging and genetics of antidepressant response to sleep deprivation: implications for drug development. Curr Pharm Des 2009; 15: 2637-2649.
47. Bunny BG, Bunney WE. Mechanisms of rapid antidepressant effects of sleep deprivation therapy: clock genes and circadian rhythms. Biol Psychiatry 2013; 73: 1164-1171.

48. Quinto C, Gellido C, Chokroverty S, et al. Posttraumatic delayed sleep phase syndrome. Neurology 2000; 54: 250-252.

49. Insel T, Cuthbert B. Toward a new classification framework for research on mental disorders. Am J Psychiatry 2010; 167: 748-751.

50. Gaspar L, van de Werken M, Johansson AS, et al. Human cellular differences in cAMP-CREB signalling correlate with light-dependent melatonin suppression and bipolar disorder. Eur J Neurosci 2014; 40: $2206-$ 2215.

51. Dallmann R, Brown SA, Gachon F. Chronopharmacology: new insights and therapeutic implications. Annu Rev Pharmacol Toxicol 2014; 54: 339-346.

52. Iranzo A, Tolosa E, Gelpi E, et al. Neurodegenerative disease status and post-mortem pathology in idiopathic rapid-eye-movement sleep behaviour disorder: an observational cohort study. Lancet Neurol 2013; 12: 443 453.

53. Kaplan KA, Harvey AG. Hypersomnia across mood disorders: a review and synthesis. Sleep Med Rev 2009; 13: $275-285$.

54. Zunzunegui C, Gao B, Cam E, et al. Sleep disturbance impairs stroke recovery in the rat. Sleep 2011; 34: 1261-1269.

55. Cornelius JR, Pittock SJ, McKeon A, et al. Sleep manifestations of voltage-gated potassium channel complex autoimmunity. Arch Neurol 2011; 68: 733-738.

56. Sabater L, Gaig C, Gelpi E, et al. A novel non-rapideye movement and rapid-eye-movement parasomnia with sleep breathing disorder associated with antibodies to IgLON5: a case series, characterisation of the antigen, and post-mortem study. Lancet Neurol 2014; 13: $575-586$.

57. Landolt H, Glatzel M, Blatter T, et al. Sleep-wake disturbances in sporadic Creutzfeldt-Jakob disease. Neurology 2006; 66: 1418-1424.

58. Morrell M, Jackson ML, Twigg GL, et al. Changes in brain morphology in patients with obstructive sleep apnoea. Thorax 2010; 65: 908-914.

59. Kumar R, Farahvar S, Ogren JA, et al. Brain putamen volume changes in newly-diagnosed patients with obstructive sleep apnea. Neuroimage Clin 2014; 4: 383 391.

60. Canessa N, Castronovo V, Cappa SF, et al. Obstructive sleep apnea: brain structural changes and neurocognitive function before and after treatment. Am J Respir Crit Care Med 2011; 183: 1419-1426.

61. Kylstra WA, Aaronson JA, Hofmann WF, et al. Neuropsychological functioning after CPAP treatment in obstructive sleep apnea: a meta-analysis. Sleep Med Rev 2013; 17: 341-347.

62. Gagnadoux F, Le Vaillant M, Goupil F. Depressive symptoms before and after long-term CPAP therapy in patients with sleep apnea. Chest 2014; 145: 1025-1031.

63. Bassetti CL. Primary and secondary neurogenic hypersomnias. Sleep Med Clin 2012; 7: 249-261.

64. Burgess CR, Scammell TE. Narcolepsy: neural mechanisms of sleepiness and cataplexy. J Neurosci 2012; 32: 12305-12311. 
65. Rolls A, Colas D, Aadamantidis A, et al. Optogenetic disruption of sleep continuity impairs memory consolidation. Proc Natl Acad Sci USA 2011; 108: 11305-11310.

66. Fronczeck R, Overeem S, Lee SY, et al. Hypocretin (orexin) loss in Parkinson's disease. Brain 2007; 130: $1577-1585$.

67. Baumann CR, Bassetti CL, Valko PP, et al. Loss of hypocretin (orexin) neurons with traumatic brain injury. Ann Neurol 2009; 66: 555-559.

68. Partinen M, Kornum BR, Plazzi G, et al. Narcolepsy as an autoimmune disease: the role of $\mathrm{H} 1 \mathrm{~N} 1$ infection and vaccination. Lancet Neurol 2014; 13: 600-613.

69. Plazzi G, Pizza F, Palaia V, et al. Complex movement disorders at disease onset in childhood narcolepsy with cataplexy. Brain 2011; 134: 3480-3492.

70. Dauvilliers Y, Bassetti C, Lammers GJ, et al. Pitolisant versus placebo or modafinil in patients with narcolepsy: a double-blind, randomised trial. Lancet Neurol 2013; 12: $1068-1075$.

71. Hübner A, Krafft A, Gadient S, et al. Characteristics and determinants of restless legs syndrome in pregnancy: a prospective study. Neurology 2013; 80: 738-742.

72. Szentkirályi A, Völzke H, Hoffmann W, et al. Multimorbidity and the risk of restless legs syndrome in 2 prospective cohort studies. Neurology 2014; 82: 2026-2033.

73. Clemens S, Rye D, Hochmann S. Restless legs syndrome: revisiting the dopamine hypothesis from the spinal cord perspective. Neurology 2006; 671: 125-130.

74. Earley CJ, Connor J, Garcia-Borreguero D, et al. Altered brain iron homeostasis and dopaminergic function in restless legs syndrome (Willis-Ekbom disease). Sleep Med 2014; 15: 1288-1301.

75. Winkelmann J, Schormair B, Licthner P, et al. Genome-wide association study of restless legs syndrome identifies common variants in three genomic regions. Nat Genet 2007; 19: 1000-1006.

76. Picchietti DL, Allen RP, Walters AS, et al. Restless legs syndrome: prevalence and impact in children and adolescents - the Peds REST study. Pediatrics 2007; 120: $253-266$.

77. Manconi M, Ferri R, Zucconi M, et al. Dissociation of periodic leg movements from arousal in restless legs syndrome. Ann Neurol 2012; 71: 834-844.

78. Allen RP, Chen C, Garcia-Borreguero D, et al. Comparison of pregabalin with pramipexole for restless legs syndrome. New Engl J Med 2014; 370: 621-631.

79. Trenkwalder C, Benes H, Grote L, et al. Prolonged release oxycodone-naloxone for treatment of severe restless legs syndrome after failure of previous treatment: a double-blind, randomised, placebo-controlled trial with an open-label extension. Lancet Neurol 2013; 12: $1141-1150$.

80. Siclari F, Khatami R, Urbaniok F, et al. Violence in sleep. Brain 2010; 133: 3494-3509.

81. Mahowald MK, Schenck CH. Dissociated states of wakefulness and sleep. Neurology 1992; 42(Suppl 6): 44-52.

82. Krueger JM, Huang YH, Rector DM, et al. Sleep: a synchrony of cell activity-driven small network states. Eur J Neurosci 2013; 38: 2199-2209.

83. Vyazovskiy VV, Olcese U, Hanlon EC, et al. Local sleep in awake rats. Nature 2011; 472: 443-447.

84. Bassetti C, Vella S, Donati F, et al. SPECT during sleepwalking. Lancet 2000; 356: 484-485.
85. Terzaghi M, Sartori I, Tassi L, et al. Evidence of dissociated arousal states during NREM parasomnia from an intracerebral neurophysiological study. Sleep 2009; 32: 409-412.

86. Nobili L, De Gennaro L, Proserpio P, et al. Local aspects of sleep: observations from intracerebral recordings in humans. Prog Brain Res 2012; 199: 219-232.

87. Gervasoni D, Lin SCL, Ribeiro S, et al. Global forebrain dynamics predict rat behavioral states and their transitions. J Neurosci 2004; 24: 11137-11147.

88. Sporns O. Network attributes for segregation and integration in the human brain. Curr Opin Neurobiol 2013; 23: $162-171$

89. Massimini M, Ferrarelli F, Sarasso S, et al. Cortical mechanisms of loss of consciousness: insight from TMS/EEG studies. Arch Ital Biol 2012; 150: 44-55.

90. Nobili L, Proserpio P, Combi R, et al. Nocturnal frontal lobe epilepsy. Curr Neurol Neurosci Rep 2014; 142: 424.

91. Silber MH, Ancoli-Israel S, Bonnet MH, et al. The visual scoring of sleep in adults. J Clin Sleep Med 2007; 3: 121-131. [Review. Erratum in: J Clin Sleep Med. 2007; 3(5): table of contents].

92. Schabus M, Dang-Vu TT, Albouy G, et al. Hemodynamic cerebral correlates of sleep spindles during human non-rapid eye movement sleep. Proc Natl Acad Sci USA 2007; 104: 13164-13169.

93. Murphy M, Riedner BA, Huber R, et al. Source modeling sleep slow waves. Proc Natl Acad Sci USA 2009; 106: $1608-1613$.

94. Dang-Vu TT, Schabus M, Desseilles M, et al. Functional neuroimaging insights into the physiology of human sleep. Sleep 2010; 33: 1589-1603.

95. van der Werf YD, Sanz-Arigita EJ, Menning S, et al. Modulating spontaneous brain activity using repetitive transcranial magnetic stimulation. BMC Neurosci 2010; 11: 145 .

96. Marshall L, Binder S. Contribution of transcranial oscillatory stimulation to research on neural networks: an emphasis on hippocampo-neocortical rhythms. Front Hum Neurosci 2013; 7: 614.

97. Adamantidis AR, Zhang F, Aravanis AM, et al. Neural substrates of awakening probed with optigenetic control of hypocretin neurons. Nature 2007; 450: 420424

98. Jego S, Glasgow SD, Gutierrez Herrera C, et al. Optogenetic identification of a rapid eye movement sleep modulatory circuit in the hypothalamus. Nat Neurosci 2013; 16: 1637-1643.

99. Vaccarino FM, Stevens HE, Kocabas A, et al. Induced pluripotent stem cells: a new tool to confront the challenge of neuropsychiatric disorders. Neuropharmacology 2011; 60: 1355-1363.

100. Liu C, Chung M. Genetics and epigenetics of circadian rhythms and their potential roles in neuropsychiatric disorders. Neurosci Bull 2015; 31: 141-159.

101. Gottlieb DJ, Hek K, Chen TH, et al. Novel loci associated with usual sleep duration: the CHARGE Consortium Genome-Wide Association Study. Mol Psychiatry 2014; Dec 2. doi: 10.1038/mp.2014.133. [Epub ahead of print].

102. Ollila HM, Kettunen J, Pietiläinen O, et al. Genomewide association study of sleep duration in the Finnish population. J Sleep Res 2014; 23: 609-618. 